\title{
Decrease of Nano-hardness at Ultra-low Indentation Depths in Copper Single Crystal
}

\author{
S. Kucharski ${ }^{1}$ - D. Jarząbek ${ }^{1}$ - A. Piątkowska ${ }^{2}$ - S. Woźniacka ${ }^{1}$
}

Received: 26 January 2015 / Accepted: 16 October 2015 /Published online: 5 November 2015

(C) The Author(s) 2015. This article is published with open access at Springerlink.com

\begin{abstract}
In the present study, we report a detailed investigation of the unusual size effect in single crystals. For the experiments we specified the hardness in single crystalline copper specimens with different orientations ((001), (011) and (111)) using Oliver-Pharr method. Our results indicates that with decreasing load, after the value of the hardness reached its maximum, it starts to decrease for very small indentation depths $(<150 \mathrm{~nm})$. For the sake of accuracy of hardness determination we have developed two AFM-based methods to evaluate contact area between tip and indented material. The proposed exact measurement of the contact area, which includes the effect of pile-up and sink-in patterns, can partially explain the strange behaviour, however, the decrease of hardness at low loads is still observed. At higher loads range the specified hardness is practically constant.
\end{abstract}

Keywords Copper $\cdot$ Single crystal $\cdot$ Nanoindentation $\cdot$ AFM . Size effect

\section{Introduction}

The mechanical properties of materials at the nanoscale have generated considerable recent research interest [1-7]. This is due to the fact that some of these properties

\section{S. Kucharski \\ skuchar@ippt.pan.pl}

1 Institute of Fundamental Technological Research, Pawińskiego 5b, 02-106 Warsaw, Poland

2 Institute of Electronic Materials and Technology, Wólczyńska 133, 01-919 Warsaw, Poland can change drastically when the dimensions of the sample decrease towards the atomistic scale. A very popular example of this effect, which has been observed in numerous nano and microindentation experiments, is an increase in hardness with decreasing depth of penetration. This phenomenon is known as the indentation size effect (ISE). Recent studies of ISE have shown that this effect for single crystals is easily observed for depths less than $1 \mu \mathrm{m}[8,9]$. Nix and Gao developed a model, based on geometrically necessary dislocations (GND), which agrees with many microindentation results [10]. According to this model, the dependence between the hardness and the indentation depth can be described as:

$H^{2}=H_{0}^{2}\left(1+\frac{h_{0}}{h}\right)$,

where $H_{O}$ is the hardness for the indefinitely large indentation depth $h$ and $h_{0}$ is a length scale which depends on the indenter geometry and the dislocation structure.

Unfortunately, Swadener et al. [11] reported that the Nix-Gao model is in a good agreement with experiments only in the case of micron scale indentations. It overestimates the hardness value for the very small indentation depths. They investigated the ISE using spherical and Berkovich indenters on the iridium alloyed with 0.3 pct $\mathrm{W}$ and $60 \mathrm{ppm}$ Th. According to them, the overestimation is due to the fact that the strong repulsion between GNDs at small indentation depths causes an expansion of the GND volume. Feng and Nix also found a similar overestimation for single crystal $\mathrm{MgO}$ [12]. They observed that there are a significant number of dislocations spreading out from the plastic zone around the indent. Hence, to relax the $\mathrm{NG}$ model's assumption that all dislocations are in the plastic 
zone, they presented a new model by defining an effective plastic zone size:

$H^{2}=H_{0}^{2}\left(1+\left(1-r e^{-h / h}\right)^{-3} \frac{h_{0}}{h}\right)$,

where $r$ and $h_{1}$ are fitting parameters.

The size effect has been analysed in a series of papers by Durst et al. [13-15], where the single crystal $\mathrm{CaF}_{2}$ as well as nanocrystaline and single crystal metals were investigated.

In their modification of the Nix-Gao model they assumed, that geometrically necessary dislocations are stored in a plastic volume (underneath the indenter) having a radius proportional to the contact radius and a proportionality factor depends on tip shape. The proposed model enables a prediction of the indentation behavior of metallic materials from uniaxial stress-strain data. The dislocation density underneath the tip was also discussed.

An analytical model of ISE, based on the maximum allowable GND density, was presented by Huang et al. [16]. An interesting approach to this problem was presented by Wo et al.[17]. In their work, the sizes of the densely dislocated zone created in Berkovich and cube-corner indents on $\mathrm{Ni}_{3} \mathrm{Al}$ at a range of indentation depths were measured directly from transmission electron microscope micrographs. The expansion of the densely dislocated zone, which they observed, was in a good agreement with the relation proposed by Feng and Nix [12]. There are also other factors, which may contribute to the difference between Eq. (1) and the experimental results gained from the Oliver-Pharr (O-P) method, such as the surface roughness [18], the longrange stress associated with GNDs [9] or the intrinsic lattice resistance [19]. An interesting method to simulate the lattice rotation and an inhomogeneous distribution of the plastic zone under the indenter tip based on the molecular dynamics was presented in [20]. The indentation size effect was also explained using flow theory of mechanism based strain gradient plasticity [21]. In [22] it has been reported that for indentations performed with spherical tips having different radii, the size effect manifested as an increase of stiffness of load-penetration curve (in scaled $P / R^{2}-h / R$ reference system) when the tip radius decreases. A detailed review of recent achievements in dealing with ISE was described in [23].

It should be noted that in the specification of hardness by means of micro/nanoindentation tests, a fundamental problem is a proper specification of the true contact area between an indenter tip and a sample's surface. This problem should be analysed more in detail when a precise evaluation of ISE is required.

The methods applied to estimate the contact area can be classified into two subcategories [24]: direct methods where the load-penetration curve is used and post-mortem methods that rely on data extracted from the residual imprint. The direct methods are widely used in the instrumented nanoindentation as they do not need the time-consuming residual imprints measurements. It is worth to note that the investigations of size effects presented above are based on direct methods. The eventual mistakes that may be made when the direct methods are applied, are a source of farther inaccuracies in the estimation of size effect.

On the other hand the post-mortem methods require advanced instruments like atomic force microscope (AFM) and scanning electron microscope (SEM). The development in scanning probe microscopy with the use of indentation tips facilitates the post mortem measurements as it allows the imaging of imprints without manipulating the sample. Nevertheless, these measurements are not so accurate as those performed with the fine AFM tip. Furthermore, there are two different objectives in the topography of residual imprint analysis in post mortem methods: the evaluation of the contact area and the identification of the material's parameters by the analysis of pile-ups patterns or cracks. In particular, the measurement of the contact area is used to verify the results of direct methods. For instance, in [24] the methodology was proposed that bases on the analysis of profiles extracted from an AFM image to specify the contact boundary of a Berkovich tip in different materials. The residual imprints generated in numerical benchmark (a conical tip) and experimental benchmark (a Berkovich tip) were investigated and a good accuracy of the proposed methodology was shown. In the case of physical experiments the "exact" contact area, considered as a reference one, was estimated through the Sneddon equation

$A_{c, S N}=\frac{\pi}{4} \frac{S^{2}}{4 \beta^{2} E_{e q}^{2}}$,

where $S$ stands for the initial unloading contact stiffness and $E_{e q}$ is estimated from the ultrasonic measurements. Furthermore, Kese and Bergman used AFM to image nanoindents made with a Berkovich indenter in soda-lime glass and then they approximated the pile-up contact perimeter as a semi-ellipse [25]. They finally achieved an empirical relationship for estimating the pile-up contact area from the contact stiffness, $S$ and the contact depth $h_{c}$. A simple AFM measurements were used to estimate the real contact area by Cao et al. [26]. In their work the effects of film thickness and substrate deformation restraint on the mechanical properties of the electron beam deposited $\mathrm{Au}$ and $\mathrm{Ag}$ films were examined by using a Berkovich indenter. To examine the residual impression in $\mathrm{Au}$ and $\mathrm{Al}$ thin films SEM and AFM were used by Zhou et al. [27]. They also applied a quantitative approach to deal with the problems with estimating the real contact area, which was 
developed by Saha and Nix [28]. It was called 'the constant modulus assumption analysis'. The basic assumption of this method is that the Young's modulus is independent of the contact area and the contact depth. The hardness can be determined from a measurement of both the load, $P$ and the contact stiffness, $S$. Zhou et al. [27] as well as Saha and Nix [28] used a Berkovich indenter in their experiments. Finally, the large review of experimental techniques for the examination of residual imprints was presented in [29]. The authors have concluded that there is a lack of consensus on the methods used to study (analyse) residual imprints generated in the indentation tests.

By contrast, in direct methods the load vs. penetration curve is analysed to determine contact depth $h_{c}$, that subsequently is used to compute the contact area $A_{c} v i a$ the tip area function $A_{c}\left(h_{c}\right)$. The area function is specified in a calibration procedure and depends on the theoretical shape of the tip and on its actual defects. The calibration methods can rely on a direct measurement of the tip's geometry or the residual imprint's geometry made in a soft material. Generally, the most common approach is to apply a special procedure, in which many indentation tests in a reference material (i.e., fused silica) are performed and the area function is evaluated on the base of the multiple $P$ - $h$ curves analysis. Different forms of area functions were discussed in the literature $[30,31]$ and similarly different equations (methods) were proposed to compute $h_{c}$ on the base of a $P$ - $h$ curve. One of the difficulties that must be overcome is the pile-up or sink-in pattern that usually occurs. This effect influences the actual contact depth. Hence, Bolshakov and Pharr used Finite Element Method (FEM) to estimate the influence of material's deformations on the Oliver and Pharr's hardness measurement method [32] and they reported that the hardness may be overestimated by up to $60 \%$ and Young's modulus by up to $16 \%$ depending on the extent of the pile-up. Although, already in 2004 Oliver and Pharr, in their review article on nanoindentation [33], discussed the problem with the pile-ups in details and concluded that the issue had to be considered as still unresolved, the problem has not been overcome until now. Nevertheless, Oliver and Pharr described some refinements and changes brought about by the improvements to the testing procedure (i.e., calibration procedure with the use of the continuous stiffness measurement on fused silica). They also conducted some FEM analysis and reported that the fundamental material properties affecting pile-up are the ratio of the effective modulus to the yield stress and the work-hardening behavior. One of the most recent approaches to this problem was presented by Cabibbo and Ricci [34]. They conducted the nanoindentation experiments with a Berkovich tip on deoxidized high phosphorus copper and then they investigated the imprints with AFM. They compared three different AFM-based methods and work of indentation analysis methods and chose one, which showed a good agreement with the literature data and the microhardness tests conducted with a Vickers indenter. Finally, they have developed this technique to make it dependent on load-penetration curve parameters instead of the AFM measurements.

In this paper we present a detailed experimental investigation of the size effect in single crystalline copper specimens having different orientations. We have observed, that for very small indentation depths $(<150 \mathrm{~nm})$, after the value of the hardness reached its maximum, it starts to decrease with the decreasing indentation depth. We called this phenomenon the inverse indentation size effect. To confirm these new non-standard, untypical results, besides the well known Oliver-Pharr method (direct method), we applied an AFM-based method (post mortem method) in our investigations of the real contact area and hardness. We also compared the values of hardness specified using both methods and we show that the Oliver-Pharr method overestimates the results at ultra low indentation depths and underestimates at greater indentation depths in single crystal copper.

\section{Experimental}

\section{Nanoindentation Setup}

A CSM Ultra Nanoindentation Tester was used to carry out the experiments presented in this paper. The device consists of an XYZ sample stage, which is used to precisely position the sample under the indenter, the atomic force microscope (AFM) and the optical microscope. At the beginning of the experiment, the sample is accurately positioned under the indenter tip. Any disturbance of the sample and of the measuring instrument (e.g., resulting from the temperature variations) are avoided due to the fact that the measuring head includes a measuring axis and a reference axis which can be controlled independently. Each axis has its own actuating means, displacement measuring system, and applied force measuring means. The load is measured by a capacitive sensor and it is applied by piezoactuator. The highest load resolution which can be achieved is $10 \mathrm{nN}$. Displacement is measured by a differential capacitive sensor with $0.001 \mathrm{~nm}$ resolution. Typical values of the thermal drift are less than $0.05 \mathrm{~nm} / \mathrm{min}$, which enables relatively long dwell time. The indentation was carried out using a Berkovich tip. After the indentation, the sample was positioned under the AFM to imagine the sample surface.

\section{Sample Preparation and Experiments}

A copper single crystal was drawn from the melt and cut precisely into samples by spark erosion and a wire saw to give (001)-, (011)-, and (111)-oriented surface planes. The 
surfaces were polished, then electro-polished, and finally carefully and rigorously cleaned with distilled water and isopropyl alcohol. Next, the electron microscopy methods were used in order to investigate the surface of the $\mathrm{Cu}$ sample. The microscope AURIGA (Zeiss) was applied. In the measurement both the secondary electrons (SE) and back scattered electrons (BSE) were detected, that enable an exploration of sample at different depths. For the SE, Everhardt-Thornley SE2 detector (for surface topography) and In-lens electron detector (for surface feature/texture analysis) were used. For the BSE, the Energy selective Backscattered (EsB) detector (suitable for clear compositional contrast) was applied. The measurement was performed with resolution of $1.5 \mathrm{~nm}$, at low primary electron beam energies, equal to $0.5 \mathrm{kV}$.

The EDS (Energy-dispersive X-ray Spectroscopy) detector of Brucker was used for preliminary chemical analysis of the bulk material. The measurements were carried out in order to ensure that the copper surface is clean. For detection of the chemical composition, the chemical compounds and structure at the surface of the sample the Electron Backscatter Diffraction (EBSD) method was applied with use of Bruker detector. Due to the electrical charge of the surface the EBSD measurements were limited to small regions, about $50 \times 2500 \mathrm{~nm}$. The depth (vertical) and the in-plane resolution of the EBSD signal were about $50-100 \mathrm{~nm}$ and $4 \mathrm{~nm}$, respectively. Thus the extremely fine structures at a surface of the sample can have been detected.

The indentation tests with a wide range of maximum indentation loads (from 0.1 to $8 \mathrm{mN}$ ) were performed on all the above orientations with the aim of investigating the influence of indentation depth on hardness. The experiments were done in the load control mode, which means that the maximum indentation load and the indentation time were set. The load was controlled by the feedback loop. The loading velocity (the increase of load in time) was equal to $50 \%$ of the maximum indentation load per minute. The choice of the unloading velocity was particularly important in these experiments due to the fact that hardness and Young's modulus are evaluated from the unloading curve. Hence, it was decided that the unloading velocity should be equal to the maximum indentation load per minute. Furthermore, the copper is a material that presents creep behaviour, hence, the $60 \mathrm{~s}$ dwell time was set. The indentation tests with the same parameters were repeated independently at least five times for the sake of statistical analysis and to avoid random inaccuracies and errors.

The experimental procedure is virtually the same as that used by other researchers. However, the application of small forces (down to $0.1 \mathrm{mN}$ ) on copper single crystal and different methods of contact area evaluation revealed an intriguing effect of a hardness decrease for very small indents.

\section{Imprints Imaging and Data Processing}

To evaluate the real contact area the AFM was used. After the indentation test, the imprint was precisely positioned under the AFM tip and scanned carefully. The horizontal resolution of the piezoactuators for the smallest imprints was $1 \mathrm{~nm} / \mathrm{pixel}$, scanning speed was $0.5 \mu \mathrm{m} \mathrm{s}^{-1}$, the signal/noise ratio was about 4 . It should be noted that due to the convolution the real horizontal resolution was not higher than $10 \mathrm{~nm}$. During the small imprints investigation the digital open feedback loop was used whereas the closed feedback loop in $\mathrm{X}$ and $\mathrm{Y}$ scanning directions was applied while bigger imprints were scanned. The resolution in the Z-range was $0.01 \mathrm{~nm}$. In order to get the row data the image processing procedures embedded in the software governing the AFM were turned off. The scanned surface topography were next post-processed using the special Hommel Map ${ }^{\circledR}$ software and the contact area was evaluated by methods described further in this paper.

\section{Results}

\section{Examination of the Surface with Electron Microscopy Technique}

The SEM images (on magnification of 40,000) reveal shallow craters homogeneously distributed on the surface of the sample - dark spots of an irregular oval shape (Fig. 1). The atomic mass of the material located in the craters is lower then the atomic mass of $\mathrm{Cu}$. The dimensions of craters and impurities spots range from 30 up to $60 \mathrm{~nm}$ and was measured directly on SEM images. The spots are ordered along regularly spaced lines. The effect of electrical charge of sample surface which was observed during the measurement indicates that the detected nano-regions are not conductive.

In the phase maps one can distinguish two phases, $\mathrm{Cu}$ and $\mathrm{Cu}_{2} \mathrm{O}$. The copper oxide $\left(\mathrm{Cu}_{2} \mathrm{O}\right)$ occurs in a form of separated small spots (clusters). The so called 'zero solution' parameter is equal to approximately $0.5 \%$ which signifies a high level of purity of the surface. The $\mathrm{CuO}$ oxide was not detected on the investigated surface. The $\mathrm{Cu}$ and $\mathrm{Cu}_{2} \mathrm{O}$ phases constitute 94.5 and $5.1 \%$ of the measured volume, respectively. By combining SEM images and EBSD maps it can be concluded that the craters are constituted by the copper oxides and the remains of the polishing treatment.

Using a special numerical code (Casino) one can estimate that at the applied low level of energy $(0.5 \mathrm{kV})$, the penetration depth of SE and BSE equals about 1.5 and $5.5 \mathrm{~nm}$ respectively. As the observed contrast was much better when one used In Lens detector (for SE), than in the case of the EsB detector, it can be concluded that some of detected BSE return from the area situated underneath of $\mathrm{Cu}_{2} \mathrm{O}$ clusters, thus the thickness of the clusters should be close to $6 \mathrm{~nm}$. On the base of analysis 
Fig. 1 SEM images of mono-Cu surface (a) the topography of surface takes with SE2 detector (b) changes in a composition chemical on the surface mono$\mathrm{Cu}$, and a contour of imprint boundary for the lowest load a)

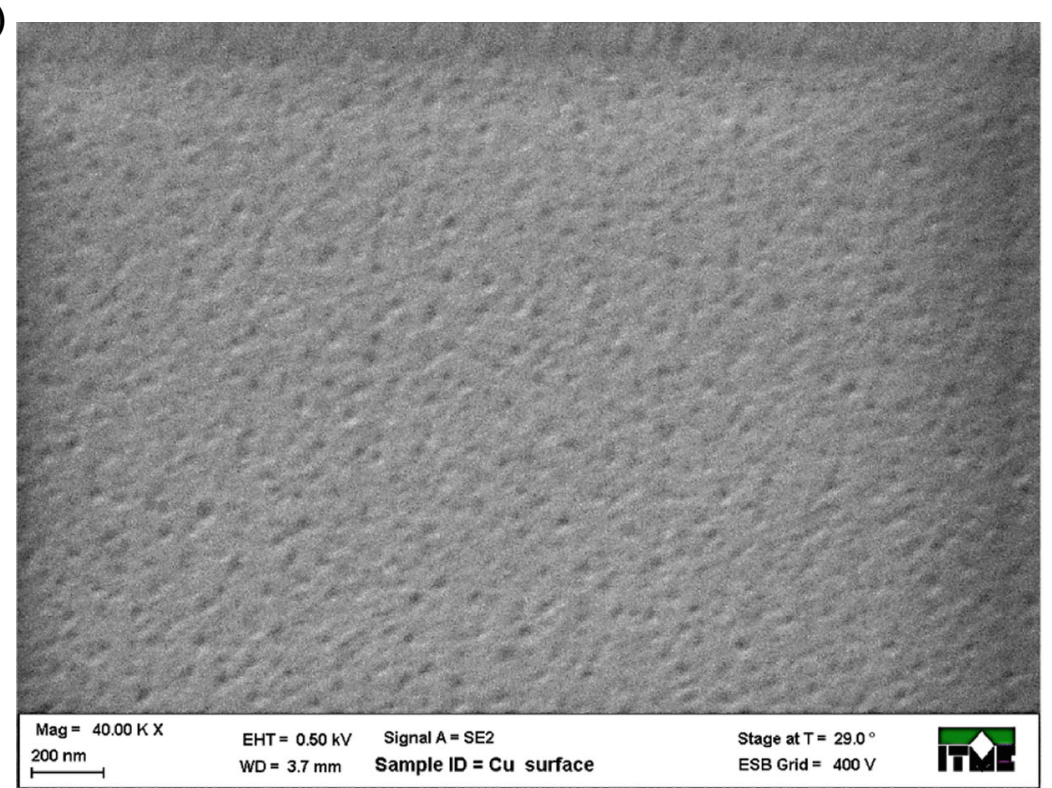

b)

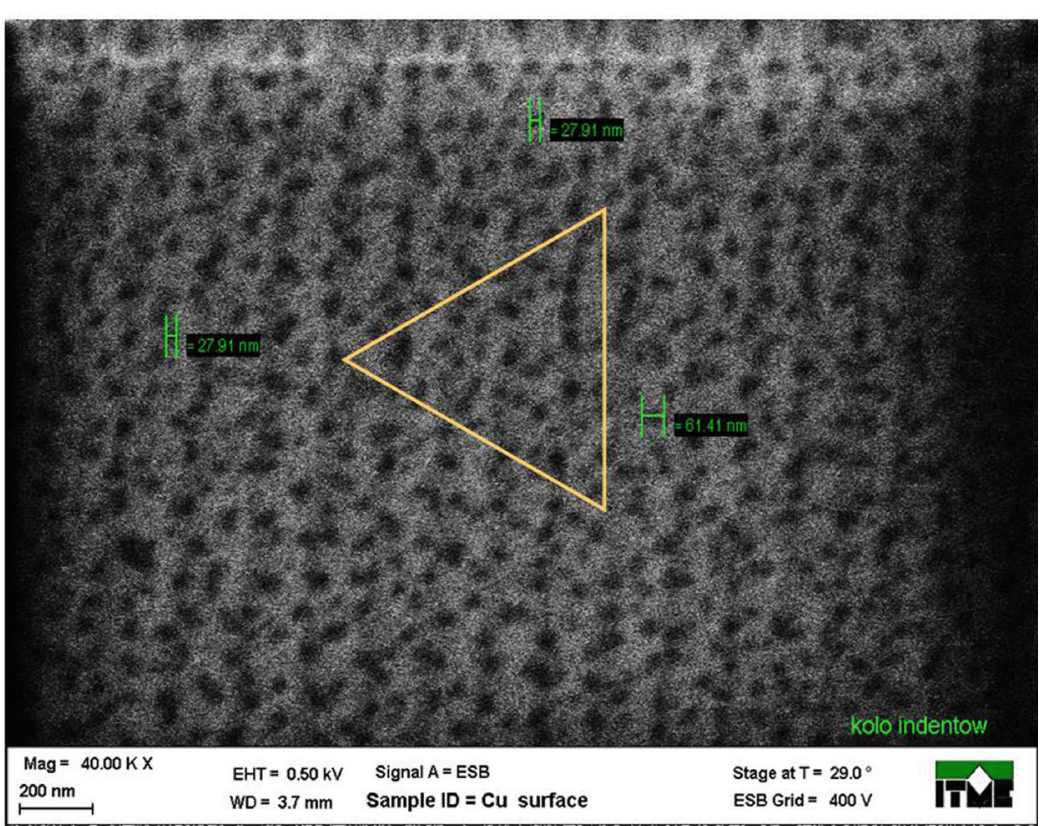

of SEM images one can carefully estimate the clusters thickness as about $8 \mathrm{~nm}$. On the other hand, the dimensions of these clusters $(30-60 \mathrm{~nm})$ are much smaller than the dimension of the smallest imprint. Therefore the presence of $\mathrm{Cu}_{2} \mathrm{O}$ clusters should not influence the results of indentation tests, even those performed with the lowest load.

\section{Preliminary Results of Hardness Measurements}

We have carried out the indentation test across the wide range of forces in order to investigate the influence of the indentation load on the hardness value. The examples of force-indentation depth curves are shown in Fig. 2. One has observed, that the creep phenomena influences a form of load-penetration curves, especially at low loads, Fig. 2(a). When the "high speed test" is performed (loading unloading in a few seconds) the unloading curve has a convex shape. Similar observation has been presented in the paper by Feng and Ngan [35], where it has been indicated that the unloading slope would become negative under some operating conditions such that the holding time at the maximum load is too short.

In such a test, a proper specification of the tangent line that is required to calculate a local derivative would be very difficult. It depends on the test speed. In the direct Olive-Pharr method, as the contact depth is calculated using analysis of unloading curve, the negative slope of this curve can 

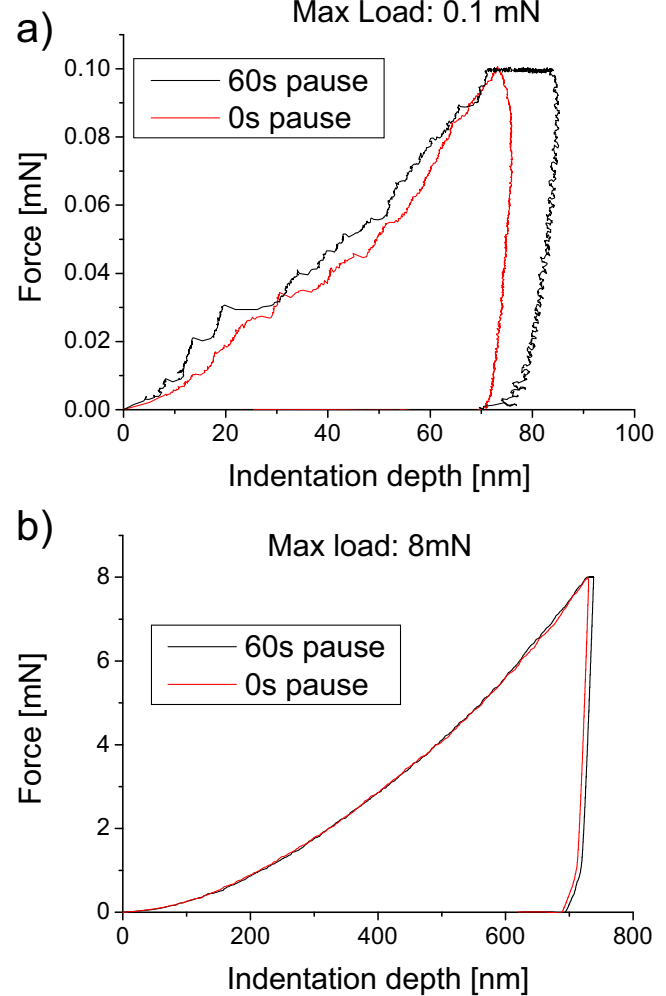

Fig. $2 P$ - $h$ curves for load parameters. (a) In the case of low loads the creep has a significant influence. The values of hardness and Young's modulus determined from the O-P method in the case of $0 \mathrm{~s}$ pause are equal to $775 \mathrm{MPa}$ and $110 \mathrm{GPa}$, respectively. On the other hand, for $60 \mathrm{~s}$ pause hardness was $536 \mathrm{MPa}$ and Young's modulus 72GPa. (b) The creep does not influence the results so strongly in the case of higher loads

influence the calculated contact area and consequently the value of hardness.

Thus we applied a sufficiently long dwell time, to avoid dependence of results on the test duration. At higher loads the results practically do not depend on the dwell time, nevertheless the $60 \mathrm{~s}$ dwell time were applied in all the cases. The evolution of the hardness with the increasing load for different indentation directions (001), (011) and close to (111) studied in the present paper is shown in Fig. 3. For each level of load many indentations were performed and a mean value has been calculated. Prior to the experiment the tip was calibrated using standard procedure. The presented results have been obtained using the standard O-P procedure implemented in the commercial software delivered with CSM instrument. The calculations of the hardness were performed using two different area functions: one was specified in the calibration procedure and the second was assumed as a nominal area function of the Berkovich tip. The comparison of both approaches enables to investigate the influence of the calibrated tip area function on the results.

One can observe, that with an increase of the load the value of hardness initially increases (the inverse indentation size effect) and reaches a maximum value for the load equal to approximately $0.8 \mathrm{mN}$. Next, for higher loads, up to the value of $8 \mathrm{mN}$, hardness continuously decreases. Similar character of hardness evolution was observed for all indentation directions. The observed tendency is different than that usually presented in the literature, where only an increase of hardness is described as the load decreases and the maximum hardness value is reached at the lowest applied load. The similar tendency is observed when the calibrated area function is replaced by nominal area, however, the hardness is shifted towards lower values for almost whole range of load. Thus, the values of calibrated area function are smaller than those of nominal one. The greatest discrepancy between both methods of hardness specification is observed at low loads.

It should be noted that only a few have observed the inverse indentation size effect. However, usually they claimed that the strange effect is due to the improper calibration of the tip bluntness. For example, Feng et. all observed a softening phenomenon in the copper single crystal indentation for indentation depths less than $250 \mathrm{~nm}$ [36]. They attribute this effect to the non-self-similar expansion of the plastic zone and/or the tip bluntness. Similar effect was reported by Durst et al. but the indentation was done with the cube corner indenter [14]. Furthermore, Liu et al. also presented the results which are in line with the results described in this paper [37]. Therefore, the effect of hardness decrease at low indentation loads was a motivation to see more in detail the specified hardness values and to analyse the influence of the experimental setup and the applied method.

\section{Discussion}

\section{Tip Shape and Area Function}

In view of a specific shape of the residual imprints and the relatively large range of the load, the problem of proper specification of the contact area has been considered. In the O-P method the area function $A_{c}(h)$ is determined using the calibration procedure, contact depth $h_{c}$ is determined from analysis of a $P$ - $h$ curve and next $A_{c}\left(h_{c}\right)$ is used to calculate hardness. The specification of the area function enables to take into account the actual tip shape.

In Fig. 4 the area function:

$$
\begin{aligned}
A_{c}\left(h_{c}\right) & =24.5 h_{c}^{2}-6.87034694 \cdot 10^{3} h_{c}+2.2199921505 \cdot 10^{5} h_{c}^{1 / 2} \\
& -5.570920137 \cdot 10^{5} h_{c}^{1 / 4} \\
& -9.015398352 \cdot 10^{5} h_{c}^{1 / 8}+1.3568625122 \cdot 10^{6} h_{c}^{1 / 16}
\end{aligned}
$$

resulting from calibration procedure is compared with the theoretical, nominal area function $A_{c T}(h)=24.5 h^{2}$ for the ideal Berkovich tip. One observes that $A_{c}(h)>A_{c T}(h)$ for very low penetration depths $(h<70 \mathrm{~nm})$ and for larger values of $h$ $(70-800 \mathrm{~nm}) A_{c}(h)<A_{c T}(h)$. 
Fig. 3 The results of the hardness evaluation. The calculations of the hardness were performed using two different area functions: one was specified in the calibration procedure $\left(\mathrm{H}_{\mathrm{OP}}\right)$ and the second was assumed as a nominal area function of the Berkovich tip $\left(\mathrm{H}_{\mathrm{NOM}}\right)$
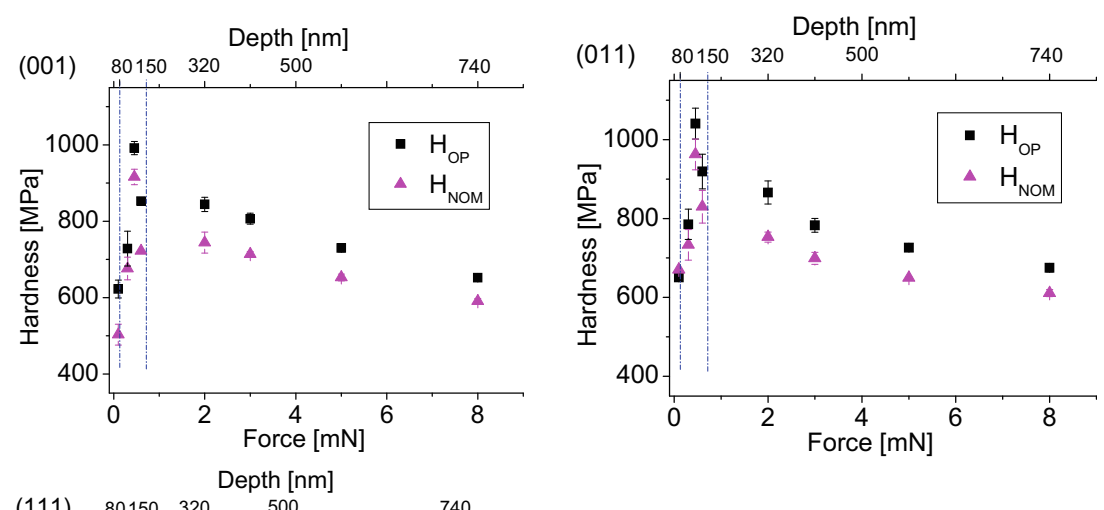

It should be noted that during the calibration procedure the indentation tests were performed in fused silica, where the penetration depth did not exceed $140 \mathrm{~nm}$. Therefore, the values of $A_{c}(h)$ for $h>140 \mathrm{~nm}$ are not reliable. They are only an extrapolation that has not been verified experimentally. The SEM and AFM micrographs, where the angles of the tip in use were measured, indicate that at large penetration depths $(h>200 \mathrm{~nm})$ the theoretical function $A_{c T}(h)$ should properly describe the change of the tip's area with increasing penetration depth. The shape of the measured tip (inclination of edges) in these micrographs corresponds to the theoretical one. Thus, it must be underlined that the calibrated area function is valid only in a limited range of penetration depth and the value of hardness specified for larger penetration depths should be checked carefully.

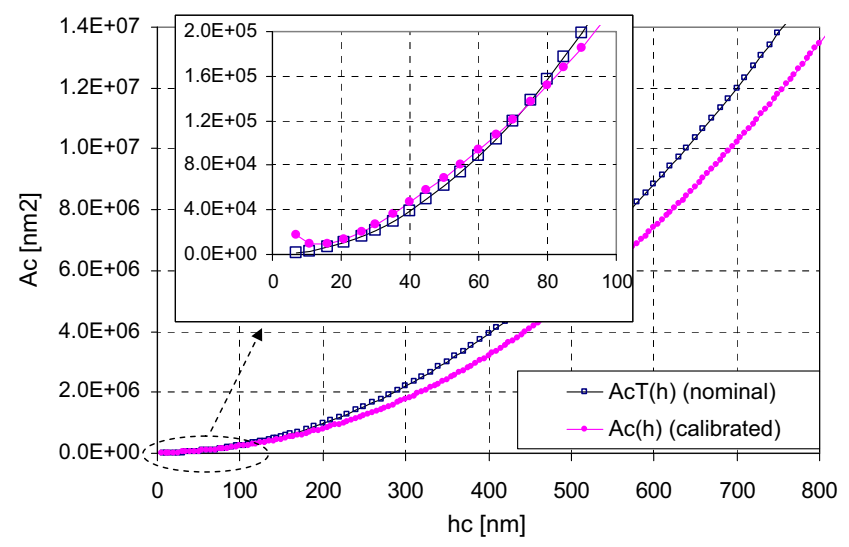

Fig. 4 Comparison of calibrated and nominal area functions
On the other hand at small penetration depths the calibrated area function is consistent with the AFM measurements of the indenter tip. The largest difference occurs for ultra-low (smaller than $15 \mathrm{~nm}$ ) penetration depths. For example, the area of tip cross-section corresponding to the $12 \mathrm{~nm}$ penetration depths can be estimated as about $8000 \mathrm{~nm}^{2}$ according to Fig. 5(b), if the influence of AFM tip radius (about $10 \mathrm{~nm}$ ) is compensated. The calibrated $A_{c}(h)$ for $h=12 \mathrm{~nm}$ that equals $8150 \mathrm{~nm}^{2}$, cf. Fig. 4 . The difference is equal to approximately $3 \%$ however it should be noted that according to Fig. 4, the real shape of the indenter for the indentation depths lower than $10 \mathrm{~nm}$ (that is close to $12 \mathrm{~nm}$ ) is not properly described by the calibration function. The calibration function below $10 \mathrm{~nm}$ starts growing which has no physical meaning. On the other hand, when the AFM measurement is concerned, one can observe a significant deviation of the tip from the pyramidal shape only below $12 \mathrm{~nm}$, Fig. 5(b). It confirms a good quality of the tip. The influence of problems with calibration function and tip bluntness on the results described in this paper are not significant due to the fact that the range of penetration depths applied in our experiments starts from approximately $80 \mathrm{~nm}$ that is six time more than $12 \mathrm{~nm}$. In this penetration range, the difference between calibrated area function and the AFM measurements was about $5 \%$ (Fig. 5(c)). It confirms a proper calibration of the area function for the lowest applied indentation depths.

Furthermore, according to the Nix and Gao model of the indentation size effect [10], the hardness of a material should not depend strongly on the depth of indentation if the material is intrinsically hard. Hence, in order to check the correctness 
a)

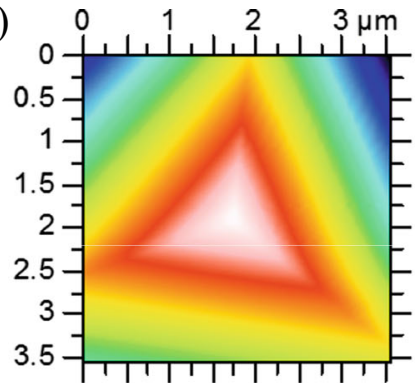

$\mu \mathrm{m}$

b)

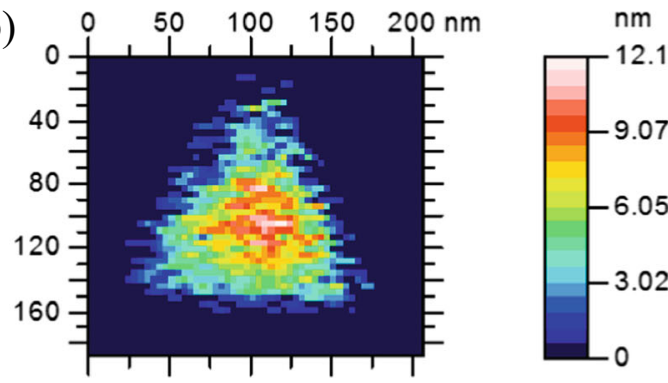

$\mathrm{nm}$

c)

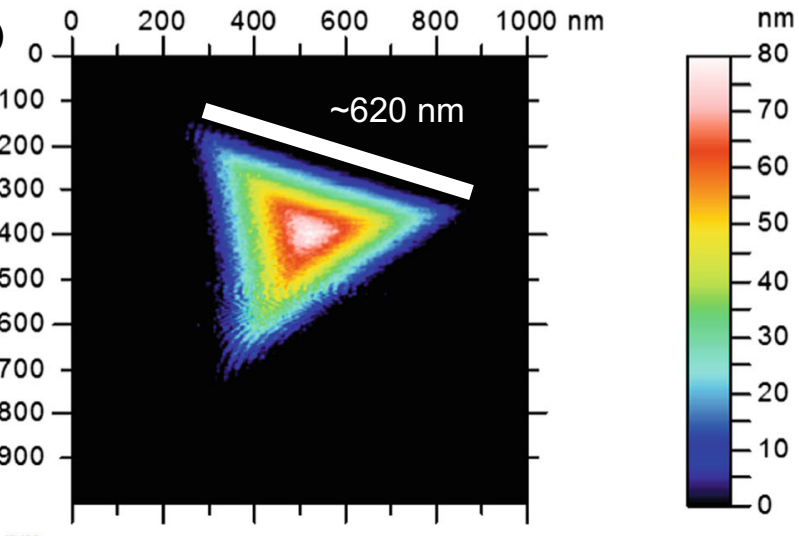

$\mathrm{nm}$

Fig. 5 AFM micrographs of the used Berkovich tip. (a) In the microscale the tip is a perfect pyramid. (b) In the nanoscale, $12 \mathrm{~nm}$ from the top of the tip, its cross-section still has a triangular shape. (c) When $h_{c}$ equals to $80 \mathrm{~nm}$ the area of the tip measured by AFM is about $0.157 \mu \mathrm{m}^{2}$ (when the AFM tip radius, about $10 \mathrm{~nm}$, is compensated!), whereas according to the calibration function it is equal to $0.152 \mu \mathrm{m}^{2}$ - the difference is about $4 \%$

of the nanoindentation setup calibration, the tests were performed on the fused silica surface. As anticipated, the hardness, even in the case of the smallest indents (a few tens of nanometers) is equal to approximately $10.5 \mathrm{GPa}$ and does not change with the indentation depth (Fig. 6). This result indicates that the calibration of the nanoindenter's tip was performed correctly. Additionally, the results gained for single crystal copper at bigger loads are in line with the results presented by other authors $[36,38]$.

\section{Comparison of the Residual and the Contact Depths}

The value of contact depth $h_{c}$ is an important parameter in the specification of contact area accordingly to the O-P procedure

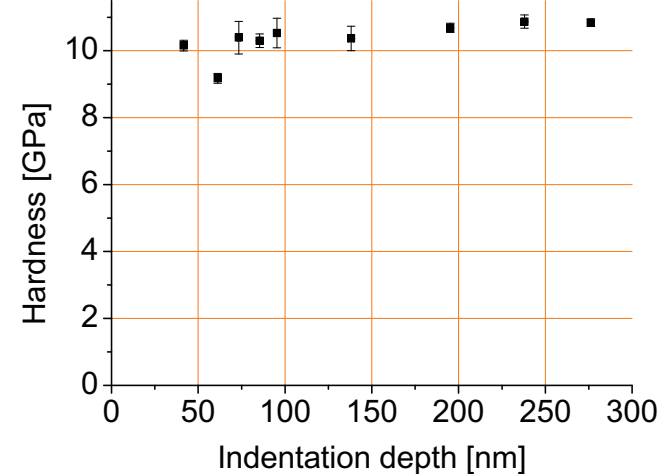

Fig. 6 Depth dependence of hardness of fused silica

as it enables to account for pile-up or sink-in pattern. In Fig. 7 the evolution of $h_{c}$ calculated using the O-P method is presented and compared with $h_{r}$ (residual depth) for different values of load. One can observe that in the whole range of loads $h_{c}>h_{r}$, thus the pile ups, visible in AFM images for the lowest loads, are taken into account, at least qualitatively, in the evaluation of the contact area. However, in the case of the highest loads, the relation $h_{c}>h_{r}$ indicates that the effect of sink-in (Fig. 8) was not taken into account in the O-P procedure.

\section{Specification of the Contact Area with Use of Post-Mortem Method}

The uncertainty in the estimation of the actual $A_{c}$ and $h_{c}$ values described above may result from an improper account for pileup and sink-in patterns that lead to an inaccurate specification of the area function. This uncertainty was a motivation to make use of the residual imprint and develop a method to estimate the true contact area in the performed nanoindentation tests.

In the proposed method, classified as a post mortem method, [28], the imprints were measured using AFM and then the Hommel Map commercial software was applied to work out the acquired images. The measured area was leveled using the least squares method - in the first step the reference plane was determined as a best fit to the whole measured area (the points in the imprint were excluded from the best fit procedure). Next the reference plane was subtracted from the raw image, thus the tilt of the initially measured area was removed. Furthermore, for the low load imprints two methods were applied in order to increase the accuracy (reliability) of the contact area estimation. It should be noted that both methods determine precisely the contact area. Due to the fact that the authors cannot state which method is better, both methods are used in this paper. Furthermore, this approach allows to confirm that the findings described here do not depend on the use of a specific post mortem method. In the first method the edge detection filter available in spatial filtering operator in Hommel Map software was used. The filter detects points having most important slope in four directions. The edge 


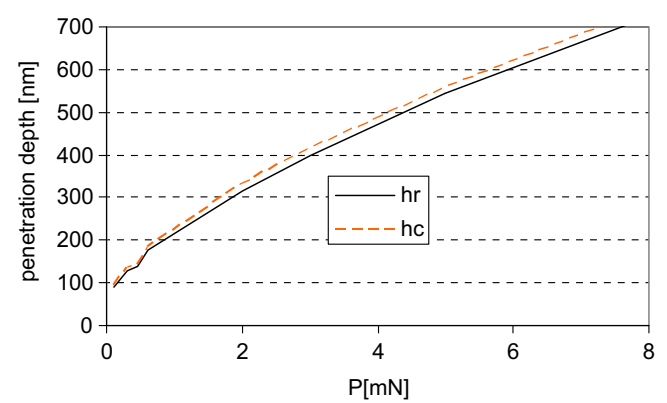

Fig. 7 Measured residual depth, and contact depth calculated with O-P method, indentation in (001) plane

detection procedure allows to detect the contact boundary of the imprint and the enclosed area can be estimated. To verify usefulness of this filter it was applied to find the contact area in indentation of fused silica. Figure 9 shows that the detected contact boundary has a convex shape and is enclosed in a triangle resulting from the tip's cross-section at the level corresponding to the measured penetration depth. Therefore, it lies below the level of an undeformed surface and its shape corresponds to the well known behaviour of fused silica. Similar shape of the contact boundary for fused silica was detected with use of the post-mortem method proposed in [24]. The measurement of residual imprints to estimate the contact area was advised also in [39].

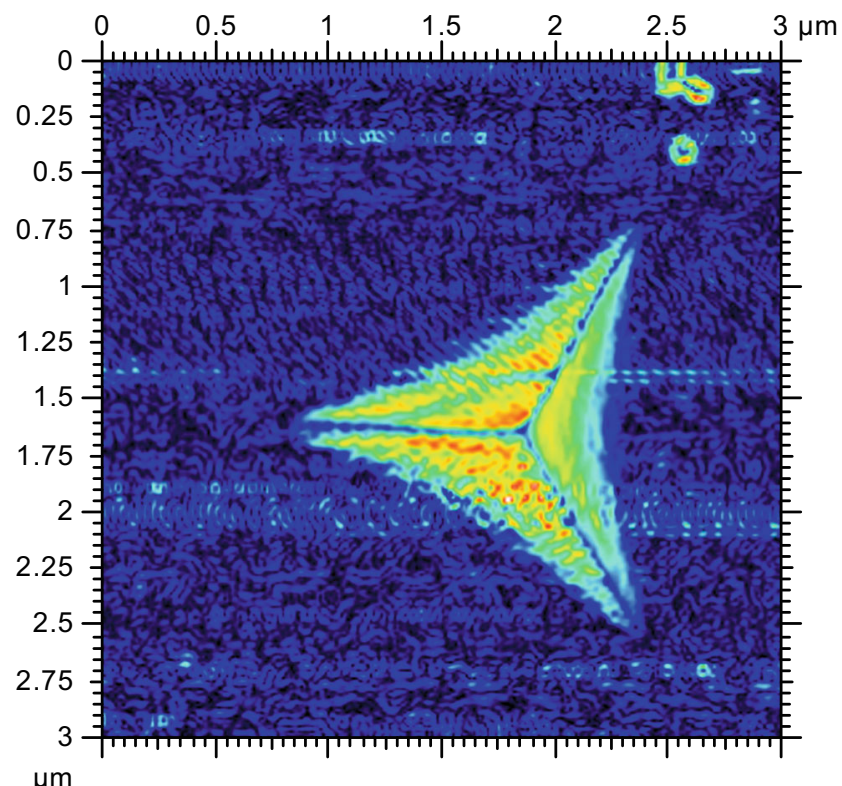

$\mu \mathrm{m}$

Fig. 9 Contact boundary detected using edge detection procedure for fused silica

The second approach consisted of fitting a plane to each of the three walls of a residual imprint. In some cases the fit parameters were further precisely adjusted manually. The points less than three standard deviations of the plane fit below the fitted plane were assumed to be in contact with the
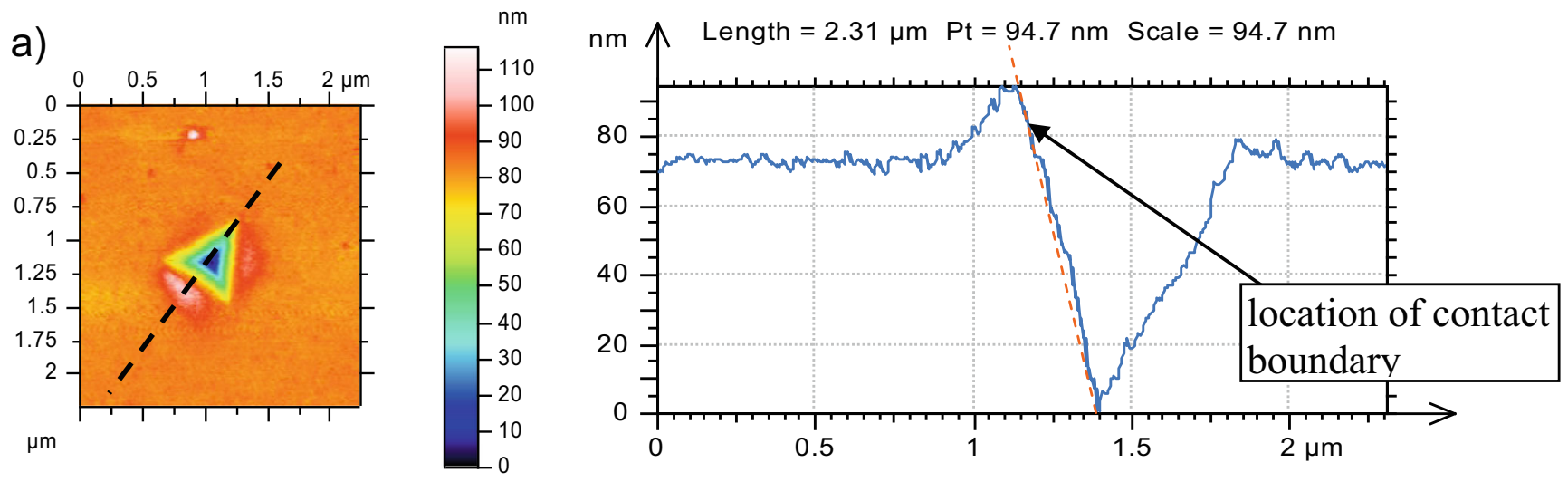

b)
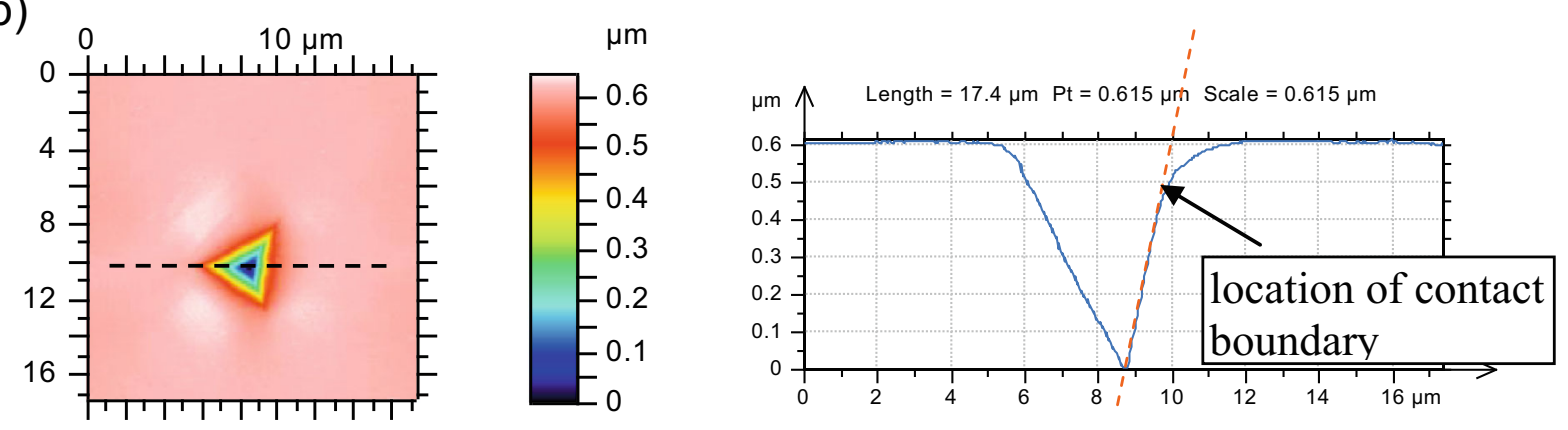

$\mu \mathrm{m}$

Fig. 8 Residual imprints for (001) plane: (a) $0.1 \mathrm{mN}$ (b) $8 \mathrm{mN}$ 
indenter. However, this approach gives good results only in the case of small indents due to the fact that for bigger indents, the walls of residual imprints are no longer plain. They are bulged in the middle.

In Fig. 10 is presented an example of both approaches in the detection of contact boundary of the imprint shown in Fig. 8(a) (low load). The contact boundary for the low loads corresponds to material that exhibits low strain hardening (pile-up patterns are visible, Fig. 1(a)). In the case of the low loads the two approaches of contact area specification described above were applied and it was shown that both methods give similar results, Fig. 11. The contact area specified using the proposed post-mortem method is slightly bigger than that resulting from estimation with O-P method. It is an effect of pile-up patterns.

In the case of the higher loads, a specific, more complex behaviour of single crystal can be observed: the sink in occurs in the vicinity of the contact boundary and hillocks are generated at a certain distance from the contact boundary, Fig. 8(b).

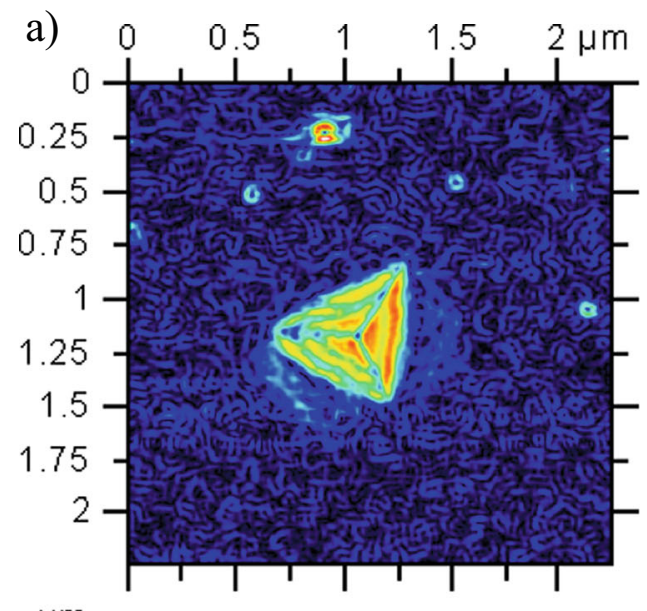

$\mu \mathrm{m}$

b)

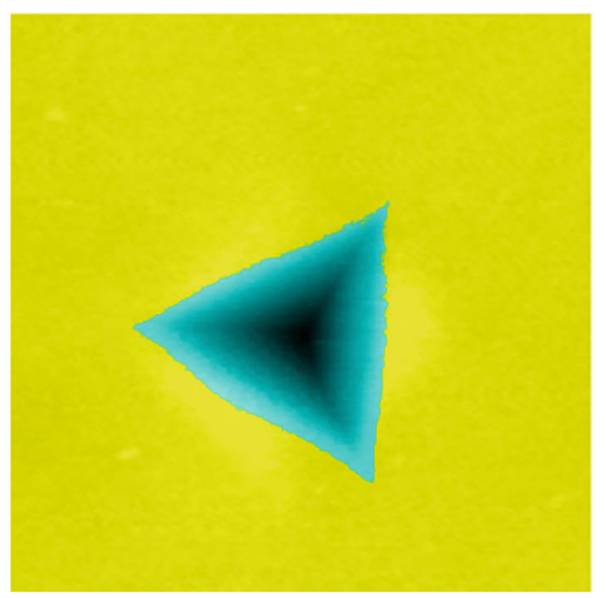

Fig. 10 Contact boundaries for imprints in (001) Cu made with low load $(0.1 \mathrm{mN})$ and detected using two methods: (a) edge detection in Hommel Map (b) plane fitting

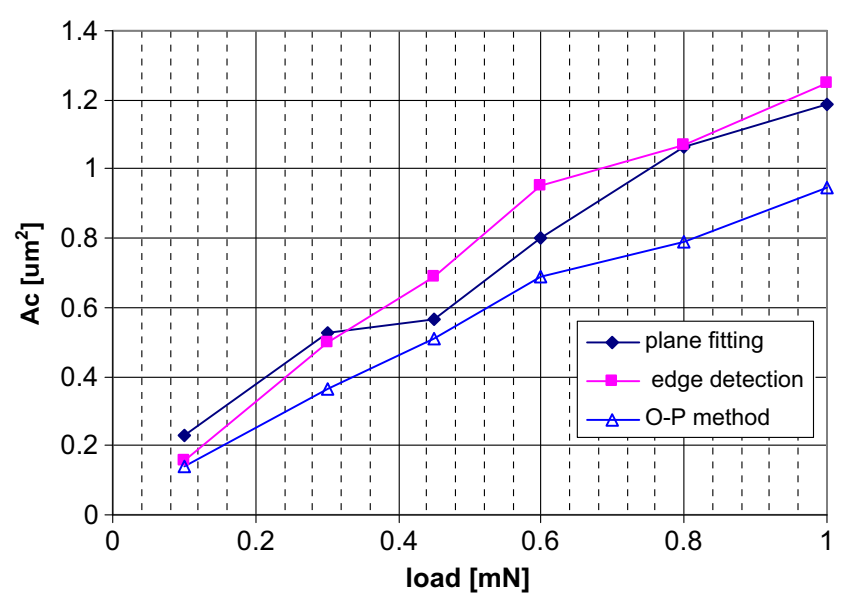

Fig. 11 Comparison of the proposed methods of specification of contact area with O-P method at low loads (110 indentation direction)

This sink-in pattern visible close to the contact boundary does not have the three-fold symmetry (tip symmetry), however, it can be qualitatively compared with a sink-in pattern generated in an isotropic material that exhibit high strain hardening e.g., fused silica. We have observed that for such response of material, where the walls of residual imprint are not flat, the first approach (edge detection, Hommel Map) presented in Fig. 12 is better suited to find the contact boundary.

In Fig. 13, the contact area specified from residual imprints using the proposed method is compared with that calculated using O-P method for the whole range of applied loads. One can observe that for $P<2 \mathrm{mN}, A_{c}$ specified with use of O-P method is smaller and for $P>2 \mathrm{mN}$ bigger than the $A_{c}$ specified from analysis of residual imprint using the proposed method.

The difference between two methods at the lowest loads results from the fact that the presence of pile-up patterns increases the contact area more evident than it is predicted by O-P method. The underestimation of contact area by the

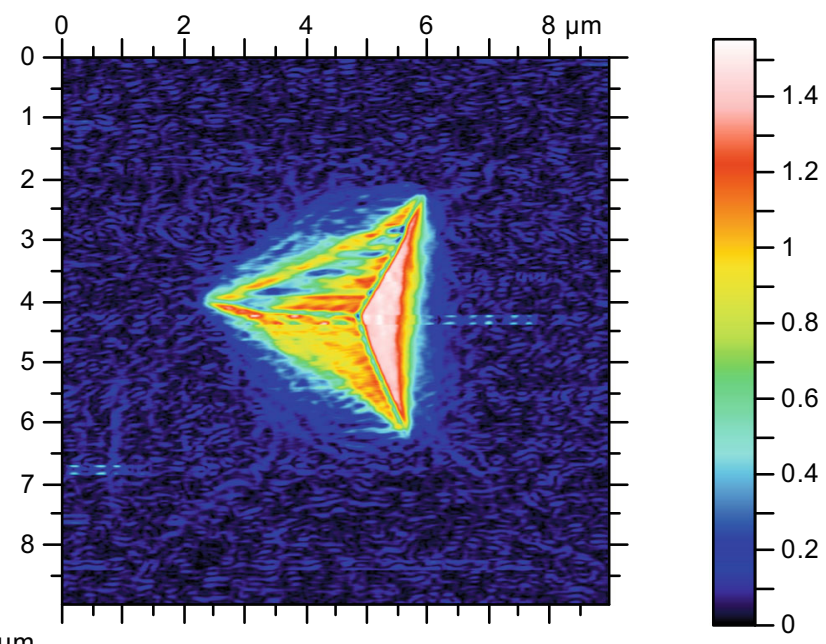

Fig. 12 Contact boundaries detected for imprints in $\mathrm{Cu}$ made with higher loads (edge detection) 


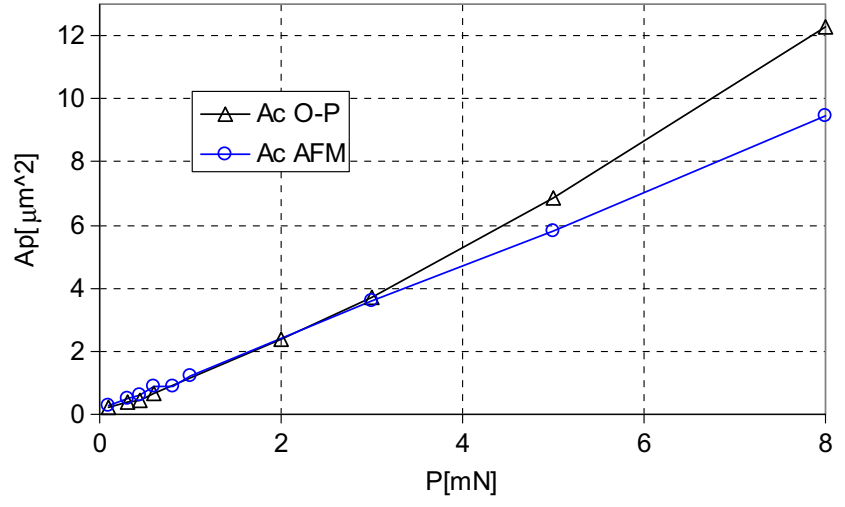

Fig. $13 A_{C P M}$ (proposed method) and $A_{C} O-P$ comparison for one indentation direction (100)

OP method is indicated also in other papers, e.g. [40]. However, this difference is smaller than that observed at larger loads. The latter results from an important effect of the reduction of the contact area due to sink-in pattern, cf. Fig. 8(b), that is not taken into account in the OP method. The evolution of the surface topography in the vicinity of residual imprints shown schematically in Fig. 8, was presented more in detail in [41]. In that work the evolution of pile-ups patterns for different directions of indentation performed with Berkovich tip and for large range of loads was studied. It was shown that at low loads the pile-up patterns are generated around the residual imprints, while in the case of higher load the sink-in pattern occurs close to the imprint boundary and relatively small hillocks are formed at a certain distance from the contact area (Fig. 8). Moreover, in the case of small imprints, the pile-up patterns are of random structure (there are one, two or three pile-ups, each of them of a different height). One can summarize, that the character of symmetry of surface topography in a vicinity of residual imprints depends on the tip symmetry at the smallest loads, whereas at higher loads it depends on the indented crystal symmetry. Such a material response influences the value of the contact area and in consequence the hardness specified in the test. It is evident that this phenomena should be taken into account when the indentation size effect is studied. Furthermore, different pile-up patterns for the lowest and the highest loads indicate the different mechanism of its creation. This different mechanism is probably also responsible for the decrease in hardness at the lowest loads shown in this paper.

\section{Comparison of Hardness Values Specified with Different Methods}

Once the contact boundaries are specified as a function of load, one can calculate the contact area and consequently the hardness in the whole range of load. Consequently, the values of hardness should change when the proposed method of specification of contact area is applied.

The values of hardness (denoted as PM-hardness) calculated using proposed method are compared with those specified with O-P method (denoted as O-P) in Fig. 14. One can observe that when the proposed method is used the evolution of hardness with an increase of load has different character than that calculated using O-P method. In both cases, for the lowest loads the hardness increases with the load. Next, for the load of about $0.8-1.2 \mathrm{mN}$, the O-P hardness reaches a local maximum and for bigger loads its value decreases, while the PM hardness reaches an approximately constant value, that next is maintained for up to the highest load. At the loads lower than about $2 \mathrm{mN}$ the O-P hardness is higher than the PM-hardness, and when the load increases an inverse relation is observed. In other words, the PM hardness is constant for large range of high loads and decreases at the lowest loads, while O-P hardness first increases and then decreases with the load. The difference of the hardness estimation with these two methods is due to the difference in estimation of the contact area by both methods. The PM hardness is slightly
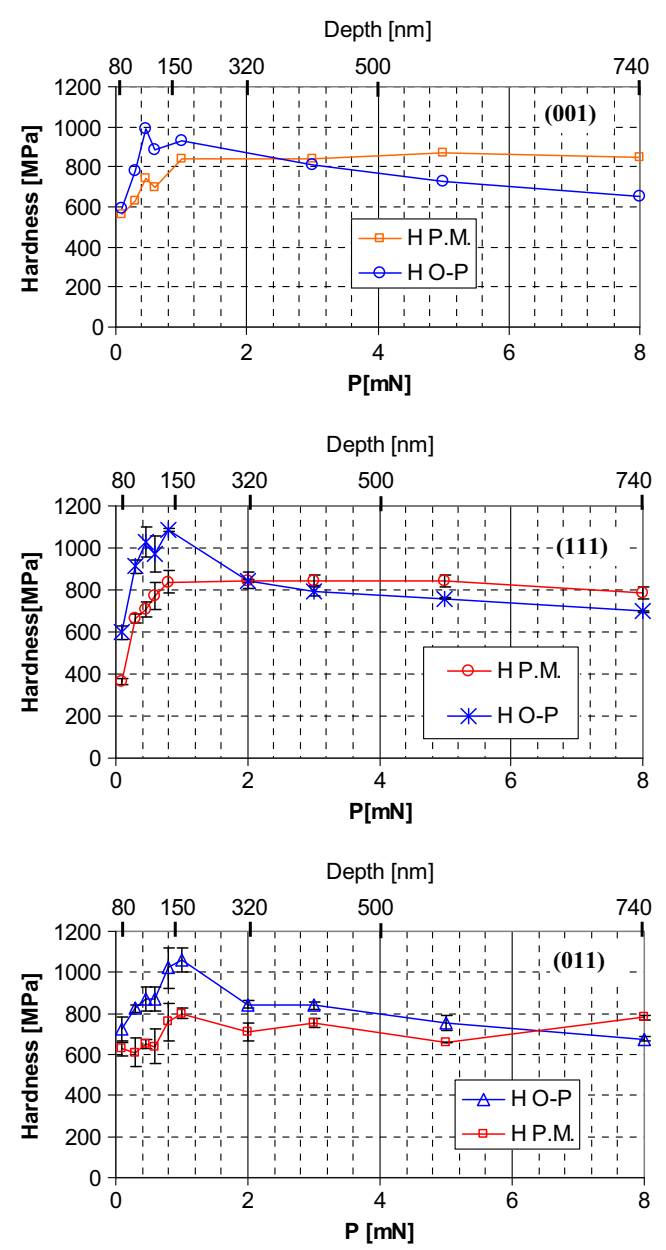

Fig. 14 The comparison between O-P hardness and PM hardness 
lower for (011) indentation direction than for the remaining (001) and (111) directions.

The proposed post-mortem specification of the contact area in the indentation tests leads to a reasonable correction of the values of the hardness. For a relatively large range of loads the value hardness is constant and it decreases for the lowest loads. We tentatively attribute this effect to different mechanisms and modes of deformation that take place at low (down to hundred nanometers) and large (few hundreds nanometers) penetration depths, as it has been mentioned at the end of "Specification of the Contact Area with Use of Post-Mortem Method" section, c.f. [41]. This effect can be explained by the fact, that for the lowest loads the motion of dislocations is rather random and is not restrained by the slip planes. On the other hand, for higher loads when the deformation depends on crystal symmetry, the dislocation motion is more difficult as it takes place only on slip planes.

The EDS measurement confirmed that the layer of the native oxide which creates on the copper surface is no thicker that $5 \mathrm{~nm}$, hence, it does not influence the results significantly - the depth of the shallowest indents was equal to $70 \mathrm{~nm}$. The presented results give a deeper insight into the indentation size effect.

To the best knowledge of the authors, the inverse indentation size effect in experiments performed with sharp tip on single crystals has not been described in details before. Admittedly according to one of latest models the nanoindentation in single crystals [42] the hardness starts decreasing with decreasing load in the lowest load range. However, it is attributed to the imperfect shape (roundness) of indenter tip at smallest depths range. The inverse size effect has been also observed recently in compression tests of nano-crystalline micro-pillars [43] and in nanoindentation tests of nanocrystaline metals [44].

\section{Conclusions}

The indentation size effect was investigated in single-crystal copper. Two approaches were applied to specify the hardness evolution with penetration depth: the well known Oliver-Pharr method and a proposed "post-mortem" method, based on a direct measurement of residual imprints. It should be emphasized that in both cases the inverse size effect was observed: at low penetration depth, down to about $150-160 \mathrm{~nm}$, the hardness decreases with the decrease of load. However the absolute value of hardness in this range of loads obtained with PM method is lower, than that calculated using O-P method.

For larger loads, when the load decreases from $8 \mathrm{mN}$ to about $1 \mathrm{mN}$ the hardness calculated with O-P method increases, that is, the "classical" indentation size effect is observed. When the PM method is used in this range of loads the hardness is larger than that specified with O-P method and it is approximately constant - the size effect is not evident.

The analysis of surface of samples with SEM reveals relatively small impurities that cannot influence the presented results of nanoindentation tests.

The different values of hardness specified with both methods is an effect of the difference between estimated areas of contact. This difference is most evident when the pile up or sink in patterns occur. Both patterns are characteristic of single crystal copper indented with Berkovich tip: at low loads the relatively large pile-ups are generated, while at highest loads the pile-ups are at certain distance from contact boundary and close to the contact the sink in patterns occur.

The observed inverse size effect can be a result of long holding time of peaks of load in our experiments. On the other hand, the same holding time was maintained for whole range of loads. It was observed that for shorter holding times at the lowest loads, the results would be rate dependent, the unloading slope would became negative. It can lead to significant errors in analysis with O-P method.

Finally, the observed phenomenon gives a deeper insight into the mechanics of deformation of single crystals. The results presented in this paper may strongly influence the researchers to seek for other examples of the inverse nanoindentation size effect and its explanation.

Acknowledgments The present research was partially supported by the Polish National Science Centre, grant NN 501067240.

Open Access This article is distributed under the terms of the Creative Commons Attribution 4.0 International License (http:// creativecommons.org/licenses/by/4.0/), which permits unrestricted use, distribution, and reproduction in any medium, provided you give appropriate credit to the original author(s) and the source, provide a link to the Creative Commons license, and indicate if changes were made.

\section{References}

1. Lotfian S, Rodriguez M, Yazzie KE, Chawla N, Llorca J, Molina-Aldareguia JM (2013) High temperature micropillar compression of $\mathrm{Al} / \mathrm{SiC}$ nanolaminates. Acta Mater 61:44394451

2. Jarzabek DM, Rymuza Z, Horiba A, Hirai Y (2011) Development of an experimental technique for testing rheological properties of ultrathin polymer films used in nanoimprint lithography. J Vac Sci Technol B 29:061603-1-061603-6

3. Kim YJ, Lee WW, Choi IC, Yoo BG, Han SM, Park HG (2013) Time-dependent nanoscale plasticity of $\mathrm{ZnO}$ nanorods. Acta Mater 61:7180-7188

4. Hayashi T, Koguchi H, Nishi N (2013) Contact analysis for anisotropic elastic materials considering surface stress and surface elasticity. J Mech Phys Solids 61:1753-1767

5. Jarzabek DM, Kaufmann AN, Schift H, Rymuza Z, Jung TA (2014) Elastic modulus and fracture strength evaluation on the nanoscale by scanning force microscope experiments. Nanotechnology 25 : 215701-215709 
6. Stelmachowski JM, Rymuza Z, Eisenmenger-Sittner C (2014) Surface properties of $\mathrm{AlCuFeB}$ coatings with focus on resolving tribological issues of nanoimprint lithography. RSC Adv 4:2947429478

7. Kravchuk A, Rymuza Z, Jarzabek D (2009) Penetration of a pyramid indenter into a multilayer coating. Int J Mater Res 100:933-935

8. Stelmashenko NA, Walls MG, Brown LM, Milman YV (1993) Microindentations on $\mathrm{W}$ and Mo oriented single crystals - an STM study. Acta Metall Mater 41:2855-2865

9. Elmustafa AA, Ananda AA, Elmahboub WM (2004) Bilinear behavior in nano and microindentation tests of fcc polycrystalline materials. J Eng Mater Technol 126:353-359

10. Nix WD, Gao HJ (1998) Indentation size effects in crystalline materials: A law for strain gradient plasticity. J Mech Phys Solids 46: 411-425

11. Swadener JG, George EP, Pharr GM (2002) The correlation of the indentation size effect measured with indenters of various shapes. $\mathrm{J}$ Mech Phys Solids 50:681-694

12. Feng G, Nix WD (2004) Indentation size effect in MgO. Scr Mater 51:599-603

13. Durst K, Backes B, Goken M (2005) Indentation size effect in metallic materials: Correcting for the size of the plastic zone. Scr Mater 52:1093-1097

14. Durst K, Backes B, Franke O, Goken M (2006) Indentation size effect in metallic materials: Modeling strength from pop-in to macroscopic hardness using geometrically necessary dislocations. Acta Mater 54:2547-2555

15. Lodes MA, Hartmaier A, Goeken M, Durst K (2011) Influence of dislocation density on the pop-in behavior and indentation size effect in $\mathrm{CaF} 2$ single crystals: Experiments and molecular dynamics simulations. Acta Mater 59:4264-4273

16. Huang Y, Zhang F, Hwang KC, Nix WD, Pharr GM, Feng G (2006) A model of size effects in nano-indentation. J Mech Phys Solids 54: $1668-1686$

17. Wo PC, Ngan AHW, Chiu YL (2006) TEM measurement of nanoindentation plastic zones in Ni3Al. Scr Mater 55:557-560

18. Zhang TY, Xu WH, Zhao MH (2004) The role of plastic deformation of rough surfaces in the size-dependent hardness. Acta Mater 52:57-68

19. Qiu X, Huang Y, Nix WD, Hwang KC, Gao H (2001) Effect of intrinsic lattice resistance in strain gradient plasticity. Acta Mater 49:3949-3958

20. Begau C, Hua J, Hartmaier A (2012) A novel approach to study dislocation density tensors and lattice rotation patterns in atomistic simulations. J Mech Phys Solids 60:711-722

21. Qiu X, Huang Y, Wei Y, Gao H, Hwang KC (2003) The flow theory of mechanism-based strain gradient plasticity. Mech Mater 35:245258

22. Kucharski S, Stupkiewicz S, Petryk H (2014) Surface Pile-Up Patterns in Indentation Testing of $\mathrm{Cu}$ Single Crystals. Exp Mech 54:957-969

23. Pharr GM, Herbert EG, Gao YF (2010) The indentation size effect: a critical examination of experimental observations and mechanistic interpretations. In: Clarke DR, Ruhle M, Zok F (eds) Annual Review of Materials Research, vol 40, pp 271-292

24. Charleux L, Keryvin V, Nivard M, Guin JP, Sangleboeuf JC, Yokoyama Y (2014) A method for measuring the contact area in instrumented indentation testing by tip scanning probe microscopy imaging. Acta Mater 70:249-258

25. Kese KO, Li ZC, Bergman B (2004) Influence of residual stress on elastic modulus and hardness of soda-lime glass measured by nanoindentation. J Mater Res 19:3109-3119
26. Cao YF, Allameh S, Nankivil D, Sethiaraj S, Otiti T, Soboyejo W (2006) Nanoindentation measurements of the mechanical properties of polycrystalline $\mathrm{Au}$ and $\mathrm{Ag}$ thin films on silicon substrates: Effects of grain size and film thickness. Mater Sci Eng A 427: 232-240

27. Zhou XY, Jiang ZD, Wang HR, Yu RX (2008) Investigation on methods for dealing with pile-up errors in evaluating the mechanical properties of thin metal films at sub-micron scale on hard substrates by nanoindentation technique. Mater Sci Eng A 488:318-332

28. Saha R, Nix WD (2002) Effects of the substrate on the determination of thin film mechanical properties by nanoindentation. Acta Mater 50:23-38

29. Marteau J, Bigerelle M, Bouvier S, Iost A (2014) Reflection on the Measurement and Use of the Topography of the Indentation Imprint. Scanning 36:115-126

30. Oliver WC, Pharr GM (1992) An improved technique for determining hardness and elastic modulus using load and displacement sensing indentation experiments. J Mater Res 7:1564-1583

31. Loubet JL, Oliver WC, Lucas BN (2000) Measurement of the loss tangent of low-density polyethylene with a nanoindentation technique. J Mater Res 15:1195-1198

32. Bolshakov A, Pharr GM (1998) Influences of pileup on the measurement of mechanical properties by load and depth sensing indentation techniques. J Mater Res 13:1049-1058

33. Oliver WC, Pharr GM (2004) Measurement of hardness and elastic modulus by instrumented indentation: Advances in understanding and refinements to methodology. J Mater Res 19:3-20

34. Cabibbo M, Ricci P (2013) True Hardness Evaluation of Bulk Metallic Materials in the Presence of Pile Up: Analytical and Enhanced Lobes Method Approaches. Metall Maters Trans A 44A:531-543

35. Feng G, Ngan AHW (2002) Effects of creep and thermal drift on modulus measurement using depth-sensing indentation. J Mater Res 17:660-668

36. Feng G, Budiman AS, Nix WD, Tamura N, Patel JR (2008) Indentation size effects in single crystal copper as revealed by synchrotron x-ray microdiffraction. J Appl Phys 104:12

37. Liu Y, Ngan AHW (2001) Depth dependence of hardness in copper single crystals measured by nanoindentation. Scr Mater 44:237241

38. Dub SN, Lim YY, Chaudhri MM (2010) Nanohardness of high purity $\mathrm{Cu}$ (111) single crystals: The effect of indenter load and prior plastic sample strain. J Appl Phys 107:043510-1043510-15

39. Fischer-Cripps AC (2006) Review of analysis and interpretation of nanoindentation test data. Surf Coat Technol 200:4153-4165

40. Sakai M (1999) The Meyer hardness: A measure for plasticity? J Mater Res 14:3630-3639

41. Kucharski S, Jarzabek D (2014) Depth Dependence of Nanoindentation Pile-Up Patterns in Copper Single Crystals. Metall Mater Trans A 45A:4997-5008

42. Qiao XG, Starink MJ, Gao N (2010) The influence of indenter tip rounding on the indentation size effect. Acta Mater 58:3690 3700

43. Zhang JY, Cui JC, Liu G, Sun J (2013) Deformation crossover in nanocrystalline $\mathrm{Zr}$ micropillars: The strongest external size. Scr Mater 68:639-642

44. Lou C, Zhang XY, Ren Y (2014) Improved strength and ductility of magnesium alloy below micro-twin lamellar structure. Mater Sci Eng 614:1-5 